This is an electronic reprint of the original article. This reprint may differ from the original in pagination and typographic detail.

Author(s): Luukko, Perttu; Helske, Jouni; Räsänen, Esa

Title: Introducing libeemd: a program package for performing the ensemble empirical mode decomposition

Year: $\quad 2016$

Version:

Please cite the original version:

Luukko, P., Helske, J., \& Räsänen, E. (2016). Introducing libeemd: a program package for performing the ensemble empirical mode decomposition. Computational Statistics, 31(2), 545-557. https://doi.org/10.1007/s00180-015-0603-9

All material supplied via JYX is protected by copyright and other intellectual property rights, and duplication or sale of all or part of any of the repository collections is not permitted, except that material may be duplicated by you for your research use or educational purposes in electronic or print form. You must obtain permission for any other use. Electronic or print copies may not be offered, whether for sale or otherwise to anyone who is not an authorised user. 


\title{
Introducing libeemd: A program package for performing the ensemble empirical mode decomposition
}

\author{
P. J. J. Luukko · J. Helske · E. Räsänen
}

Received: date / Accepted: date

\begin{abstract}
The ensemble empirical mode decomposition (EEMD) and its complete variant (CEEMDAN) are adaptive, noise-assisted data analysis methods that improve on the ordinary empirical mode decomposition (EMD). All these methods decompose possibly nonlinear and/or nonstationary time series data into a finite amount of components separated by instantaneous frequencies. This decomposition provides a powerful method to look into the different processes behind a given time series data, and provides a way to separate short time-scale events from a general trend.

We present a free software implementation of EMD, EEMD and CEEMDAN and give an overview of the EMD methodology and the algorithms used in the decomposition. We release our implementation, libeemd, with the aim of providing a user-friendly, fast, stable, well-documented and easily extensible EEMD library for anyone interested in using (E)EMD in the analysis of time series data. While written in $\mathrm{C}$ for numerical efficiency, our implementation includes interfaces to the Python and $\mathrm{R}$ languages, and interfaces to other languages are straightforward.
\end{abstract}

Keywords Hilbert-Huang transform · Intrinsic mode function · Time series analysis $\cdot$ Adaptive data analysis $\cdot$ Noise-assisted data analysis $\cdot$ Detrending

\author{
P. J. J. Luukko \\ Nanoscience Center, University of Jyväskylä, FI-40014, Finland \\ E-mail: perttu.luukko@iki.fi \\ J. Helske \\ Department of Mathematics and Statistics, University of Jyväskylä, FI-40014, Finland \\ E. Räsänen \\ Department of Physics, Tampere University of Technology, FI-33101, Finland
}




\section{Introduction}

Empirical mode decomposition (EMD) is a method for decomposing and analyzing time series data which may be nonlinear and/or nonstationary (Huang et al, 1998). The EMD procedure decomposes the input signal (the time series data) into a collection of intrinsic mode functions (IMFs), which are simple oscillatory modes with meaningful instantaneous frequencies, and a residual trend. When combined with Hilbert spectrum analysis (HSA) to extract the instantaneous frequencies of the IMFs, EMD becomes a powerful tool for analyzing and characterizing the underlying processes behind any given time series data. The combination of EMD with HSA is often called the Hilbert-Huang transform (HHT) (Huang and Shen, 2005).

While originally a data analysis tool for Earth sciences (see, e.g., Huang and $\mathrm{Wu}$ (2008) and references therein), the generality of EMD and HHT has resulted in applications in fields ranging from medicine (Pachori and Bajaj, 2011) to finance (Zhang et al, 2008). It can be used for speech recognition (Huang and Pan, 2006) and detrending energy-level spectra of quantum systems (Morales et al, 2011). Given its versatility, it is likely that EMD will continue to find new problems to solve in all fields of science dealing with empirical data. Hopefully future studies will also give EMD a more well-understood mathematical foundation, which is still somewhat lacking (Huang and $\mathrm{Wu}, 2008$ ).

The original EMD algorithm has also resulted in many derivative algorithms which improve on the original design and adapt the algorithm to more specific uses. A recent improvement from the original authors of EMD is ensemble empirical mode decomposition (EEMD) in which additional noise is used to better separate different frequency scales into different IMFs without using subjective selection criteria (Wu and Huang, 2009). The original EEMD method is not a complete decomposition, since the original signal cannot be exactly recovered by adding together its EEMD components. Instead, a more recent variant CEEMDAN (Complete EEMD with Adaptive Noise) by Torres et al (2011) achieves completeness while improving the algorithm's robustness against noisy input signals (Colominas et al, 2012).

The purpose of this work is to provide a fast, generic, well-documented and easily accessible implementation of EMD, EEMD and CEEMDAN for anyone interested in using them in their research. Our code library will also hopefully serve as a basis for implementing existing and future derivatives of EMD. To foster the use and further development of our program we release it under a free software license. The source code of our program can be freely downloaded from https://bitbucket.org/luukko/libeemd. 


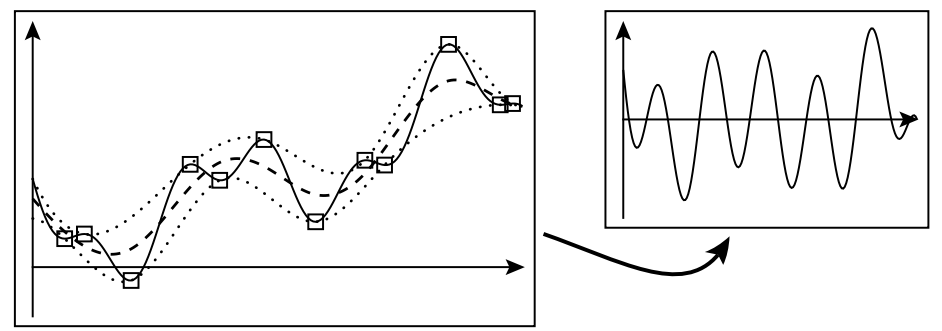

Fig. 1 The sifting procedure isolates oscillations around the "local mean" of the signal. First, on the left panel, local extrema (open squares) of the signal (solid line) are located. Then, upper and lower envelopes (dotted lines) of the signal are formed by connecting the local maxima and minima, respectively, with a spline function. The mean of these envelopes (dashed line) is designated as the local mean of the signal. By subtracting the local mean from the original signal, oscillations with high local frequency are isolated into a new, simpler and more symmetric signal, shown on the right panel.

\section{Algorithms}

\subsection{Empirical mode decomposition}

The basics of the empirical mode decomposition algorithm are documented well in the literature (Huang et al, 1998; Huang and Wu, 2008; Dätig and Schlurmann, 2004), but for completeness we recapitulate the general idea. The target of the EMD procedure is to decompose a signal - in the spirit of the Fourier series - into a sum of simple components. In contrast to the Fourier series, these components are not required to be simple sinusoidal functions, but they are required to have meaningful local frequencies. The components are called intrinsic mode functions (IMFs), and the requirement of a meaningful local frequency is enforced by requiring two conditions (Huang et al, 1998): (1) the number of zero crossings and the number of local extrema of the function must differ by at most one and (2) the "local mean" of the function is zero. What is meant by a local mean in this case is elaborated below.

At the heart of EMD is the sifting procedure, which extracts the simple oscillatory components (the IMFs) from the original signal. First, upper and lower envelopes of the signal are computed by finding the local extrema of the signal. To construct the upper (lower) envelope, the local maxima (minima) are connected by a smooth interpolation - typically a cubic spline. Then the mean of these envelopes is designated as a "local mean" of the signal, which can be used as a reference that separates the lower frequency oscillations in the signal (the part included in the local mean) from the highest frequency oscillations (the oscillations around the local mean). This procedure is shown schematically in Fig. 1. Note that the separation into "high frequency" and "low frequency" is now based purely on the rapidity of oscillations in the original signal. Also, what is considered high frequency in one part of the signal can be low frequency in another part, since the local mean can oscillate wildly in some part of the data and change slowly in other parts. 
By subtracting the local mean from the original signal, we can separate the high (local) frequency oscillations from the rest of the signal. However, this subtraction can create new local extrema, foiling the requirements set for an IMF. To actually recover the highest frequency IMF component, the sifting procedure is applied again and again, until some stopping criterion is fulfilled and we are left with a sufficiently pure IMF. The choices of stopping criteria are discussed further in Sec. 2.5. After the first IMF is obtained, it can be subtracted from the original signal, and the procedure outlined above can be used to extract the IMF with the second-highest local frequency. This can be repeated until the residual signal is monotonous, and no further IMFs can be extracted. This residual, possibly together with the lowest frequency IMFs, can be used to represent the intrinsic trend of the data. Detecting and removing such a trend is alone a useful application of EMD for several purposes ( $\mathrm{Wu}$ et al, 2007; Morales et al, 2011).

EMD separates different frequency scales of the signal into separate IMFs, but it is not guaranteed that - when analyzing a data from some natural process - each IMF represents a physical time scale of the process. Often ranges of IMFs need to be added together to extract information pertaining to a single natural time scale (Wu and Huang, 2009), and some IMF components may represent the properties of measurement noise instead of the underlying physical process. To assist in selecting the IMFs with a physical meaning $\mathrm{Wu}$ and Huang (2004) have constructed a statistical significance test which compares the IMFs against a null hypothesis of white noise.

\subsection{Detection of extrema and zero slopes}

The local extrema of the data sequence, which are needed to form the upper and lower envelopes, can of course be found by simply comparing consecutive data points. However, some care is needed in the handling of zero slopes, i.e., stretches of strictly equal data points in the sequence. The reference EEMD library by $\mathrm{Wu}$ and Huang (2009) considers consecutive equal data points to be both maxima and minima, which causes the upper and lower envelopes to meet at these points. This is probably an unwanted feature since, e.g., a small deviation at the highest point of a large oscillation can cause the topmost data points to be equal, causing in turn the lower envelope to jump sharply to meet the upper envelope at the local maximum, as illustrated in Fig. 2.

Naturally, exactly equal data points in the original signal are likely to be a result of artificial data or low sample accuracy, but such zero slopes can also occur in the intermediate steps of the EMD procedure, especially when the number of samples is low.

To ameliorate these issues with zero slopes, we choose a different convention for the extrema detection. For a point to be considered a local maximum, the slope before the data point has to be strictly positive, and the slope after the data point strictly negative. However, if there is a region of zero slope between the positive and the negative slopes, the center of the zero slope 


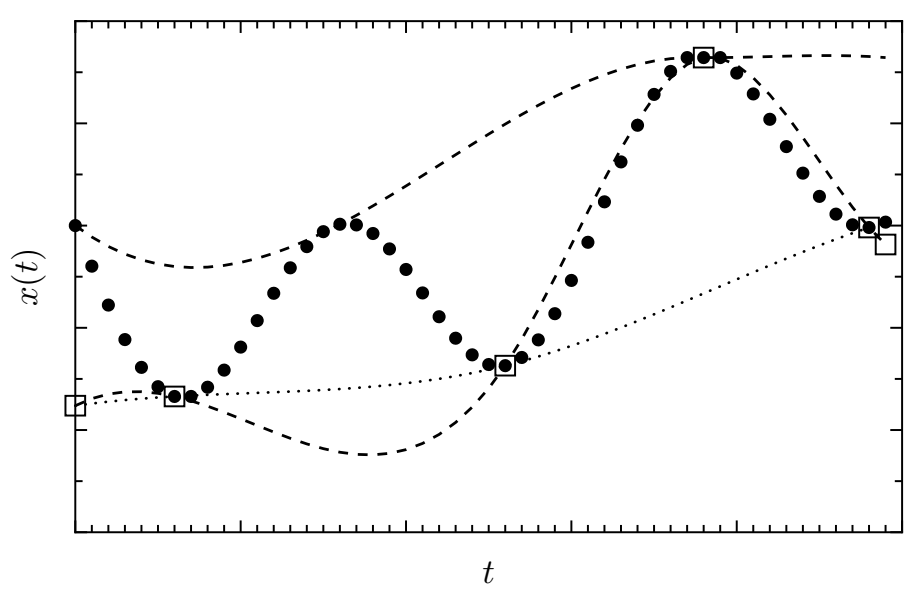

Fig. 2 Plot highlighting the problem caused by equal consecutive data points in the reference EEMD implementation by Wu and Huang (2009). The filled circles show an artificial signal $x(t)$, in which three data points near the top of an oscillation happen to be equal within sample precision. The squares and the dashed lines show the local minima and the envelope splines, respectively, as found by the reference EEMD implementation. Because the middle one of the equal data points is considered both a maximum and a minimum, the lower envelope spline shoots up sharply, no longer representing a good lower envelope for the signal. The lower envelope produced by libeemd is shown as a dotted line

region is designated as a single maximum. Likewise, valleys with a flat bottom are considered to have a single minimum at the center of the flat region. This convention is also used by another implementation by Torres et al (2011).

\subsection{Spline envelopes}

There are several ways to interpolate the local extrema to form the upper and lower envelopes. A cubic spline is considered by most to be the best trade-off between smooth envelopes and simplicity (Huang and $\mathrm{Wu}, 2008$ ), but there are several different kinds of cubic splines. In their review Dätig and Schlurmann (2004) consider the so-called natural cubic spline the best choice for data from water waves. We adopted the convention used by the reference EEMD library by Wu and Huang (2009) and used cubic splines with the "nota-knot" end conditions. These splines are somewhat more computationally intensive to calculate, but unlike with the "natural" end conditions, the splines are not required to have zero curvature at the ends of the data, which we consider to be an artificial limitation. For computing the splines we use the algorithm described by Engeln-Müllges and Uhlig (1996). As the reference EEMD implementation, our code falls back to linear interpolation (for $N=2$ ) or polynomial interpolation (for $N=3$ ) if the number of extrema $N$ is too small for a cubic spline.

More recently effort has been made into using B-splines for the envelopes to establish a firmer mathematical basis for EMD (Chen et al, 2006). In addi- 
tion, piecewise cubic Hermite interpolation polynomials (PCHIPs) have been suggested to replace the usual cubic splines to better interpolate highly nonstationary signals (Shulin et al, 2007). Both of these are considered to be useful alternatives to be added in the future to libeemd.

\subsection{End effects}

While cubic splines are very good at interpolating data at the interior of the data sequence, they perform worse at the ends of the data. This is of course a problem shared by interpolation in general - at the ends of the data the interpolation algorithm can only work with the "neighborhood" of data points on one side of the end, since the other side is missing. This turns the interpolation problem into an extrapolation one - how to predict how the data would behave before the start of the sequence? If left unattended, the errors of the spline interpolation can cause the ends of the computed IMFs to become corrupted, and as the sifting procedure continues these errors propagate to the interior to the data.

Several ways have been proposed to mitigate the end effects by adding artificial extrema to the ends of the data, such as simple wave forms defined by the extrema near the end (Huang et al, 1998). We have adopted the method described by $\mathrm{Wu}$ and Huang (2009), where additional extrema are added to the ends of the data by linear extrapolation of the previous two extrema. However, if the extrapolated extremum is less extremal than the last data point, the value of the last data point is used as an additional extremum instead. This method successfully reduces the end effects while avoiding the possible complications of more complex data extrapolation.

\subsection{Stopping criteria}

Many ways to decide how many times a signal is sifted to produce a single IMF have been proposed. The original algorithm by Huang et al (1998) used a Cauchy-like criterion, in which the sifting is stopped when the normalized square difference of results from consecutive iterations is sufficiently small. However, this method is vulnerable to sifting the signal too much so that it becomes a frequency-modulated signal only and all amplitude variation is lost. It is also not guaranteed that the final result will fulfill the requirements of an IMF. Therefore Huang et al (1999) proposed a simpler stopping criteria, in which iteration is stopped when the number of zero crossings and extrema differ at most by one and that these numbers stay the same for $S$ consecutive iterations. This criterion was extensively studied by Huang et al (2003) and the optimal range for the $S$-number was found to be from 3 to 8 . Our code supports $^{1}$ using this stopping criterion for the siftings with a default value

\footnotetext{
1 In rare cases the finite precision of computer arithmetic can cause the number of zero crossings or extrema to get stuck oscillating between two consecutive numbers. To avoid
} 
of $S=4$. In addition, a maximum number of total iterations can be set to prevent oversifting. By only setting the maximum number of iterations, EMD can be performed with a fixed number of iterations, which is sometimes preferred as described by $\mathrm{Wu}$ and Huang (2009).

\subsection{Ensemble EMD and CEEMDAN}

A recently demonstrated improved variant of the EMD method is Ensemble EMD (EEMD), in which EMD is performed on an ensemble of initial signals, each perturbed by low-amplitude white noise (Wu and Huang, 2009). The noise helps the sifting process to avoid mode mixing and to provide more robust and physically meaningful IMFs. In the end the average of the results is designated as the true final result, and thus the direct effect of the noise is canceled out. Computing the EMD of a large ensemble of signals is computationally more intensive, but this difference in computation time can be reduced significantly since the separate ensemble members can be computed in parallel.

Because the added noise does not completely cancel out in the averaging process for any finite ensemble size, EEMD is no longer a strictly complete decomposition. This issue has been fixed in a EEMD variant called CEEMDAN (Torres et al, 2011). In CEEMDAN, the averaging over all ensemble members is carried separately for each IMF component. By changing the order of averaging over the ensemble and extracting the next IMF, at each point of the decomposition procedure the current residual together with the already extracted IMFs sums exactly (or up to numerical precision) to the original signal. This small change also seems to improve the algorithm's efficiency in recovering an underlying tone from an already noisy input signal (Colominas et al, 2012).

\section{Implementation details}

\subsection{Low-level C library}

The low-level computational methods of libeemd are written in standardscompliant C99 for numerical efficiency and portability. The $\mathrm{C}$ interface to libeemd is documented in the header file eemd.h distributed with libeemd. All the methods for computing EMD, EEMD or CEEMDAN can be used directly from $\mathrm{C}$, but we focus on providing interfaces so that libeemd can be accessed from more high-level languages which are better suited for data analysis. We provide complete interfaces to the Python and R languages, which are excellent modern languages for data analysis tools and other scientific software. In the future, interfaces to other languages will be considered.

an endless loop or extreme oversifting in this case our implementation relaxes the latter condition so that a case where only one of the numbers changes by 1 is still considered stable. This change does not affect the normal operation of EMD. 
For random number generation and basic linear algebra we use routines provided by the GNU Scientific Library (GSL) (Galassi et al, 2009). For faster EEMD and CEEMDAN computations with large ensembles the separate ensemble members are handled in parallel with OpenMP. More elaborate parallelization schemes, which benefit large, multidimensional EEMD calculations, also exist in the literature (Chang et al, 2011). The library can also be compiled in single-thread mode if parallelization is not desired.

\subsection{Python interface}

The libeemd distribution includes a Python interface called pyeemd. This interface allows the routines in libeemd to be called directly from Python, using standard Numpy (Oliphant, 2007) arrays for input and output data. Via pyeemd the routines provided by libeemd can be accessed with a minimal amount of overhead code. To give a short example, if we have an input signal stored in a 1D Numpy array (or some other Python sequence type) input, we can decompose it with EEMD using the code:

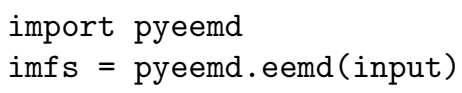

Now imfs is a $M \times N$ array, where $N$ is the length of input, and the IMFs it decomposed to are stored in the $M$ rows of imfs, the last row being the residual. The individual IMFs can then be manipulated easily with the numerous arithmetic tools provided by Numpy. For completing the Hilbert-Huang transform, the Hilbert transformation routine provided by the Scipy package (scipy.fftpack.hilbert) can be used. The IMFs can be visualized by any of the several plotting libraries available to Python, but we also provide a simple helper routine (pyeemd.utils.plot_imfs) for quick visualization of the results.

The stopping criterion for the decomposition can be set by using the optional parameters S_number and num_siftings, corresponding to the $S$ number criterion and a maximum number of siftings, respectively, as described in Sec. 2.5. The default values are $\mathbf{S}$ number $=4$ and num_siftings $=50$. Other optional parameters that can be used to influence the EEMD decomposition are ensemble_size and noise_strength. The parameter ensemble_size is the size of the EEMD ensemble and it defaults to 250. The magnitude of the Gaussian white noise added to the input in EEMD is controlled by noise_strength, which represents the standard deviation of the Gaussian random numbers used as the noise, relative to the standard deviation of the input. It defaults to setting the noise to have 0.2 times the standard deviation of the signal. This value was suggested by $\mathrm{Wu}$ and Huang (2009) and shown to be a good default value for many cases by Colominas et al (2012). Computing a simple EMD decomposition can be done by setting ensemble_size to 1 and noise_strength to 0 , but we also provide as a shortcut the routine pyeemd.emd.

The pyeemd distribution also includes unit tests which can be used to ensure that the code is working as intended. These tests can also be used to 
ensure that our code reproduces the results obtained by the reference EEMD implementation, except for the case of equal data points described in Sec. 2.2. For more documentation of the use and internals of pyeemd please see the documentation files and source code distributed with pyeemd, and the online documentation at http://pyeemd.readthedocs.org/.

\section{$3.3 \mathrm{R}$ interface}

In addition to a Python interface, we have written an interface to $\mathrm{R}$ in a form of a complete $\mathrm{R}$ package Rlibeemd. A stable version of the package is available at $\mathrm{CRAN}^{2}$, and the latest development version is also available at GitHub ${ }^{3}$. With help of Rcpp package (Eddelbuettel and François, 2011; Eddelbuettel, 2013) the libeemd $C$ library is integrated into $R$ with minimal overhead. Input data for decomposition can be a numeric vector or an object which can be coerced to such. Output data is converted to a time series object of class mts for easier plotting and further analysis. We present a short example using the UK gas consumption dataset which is a part of the base $\mathrm{R}$ dataset collection. We perform a CEEMDAN decomposition for logarithmic consumption using default values of the ceemdan function, and plot the resulting IMFs:

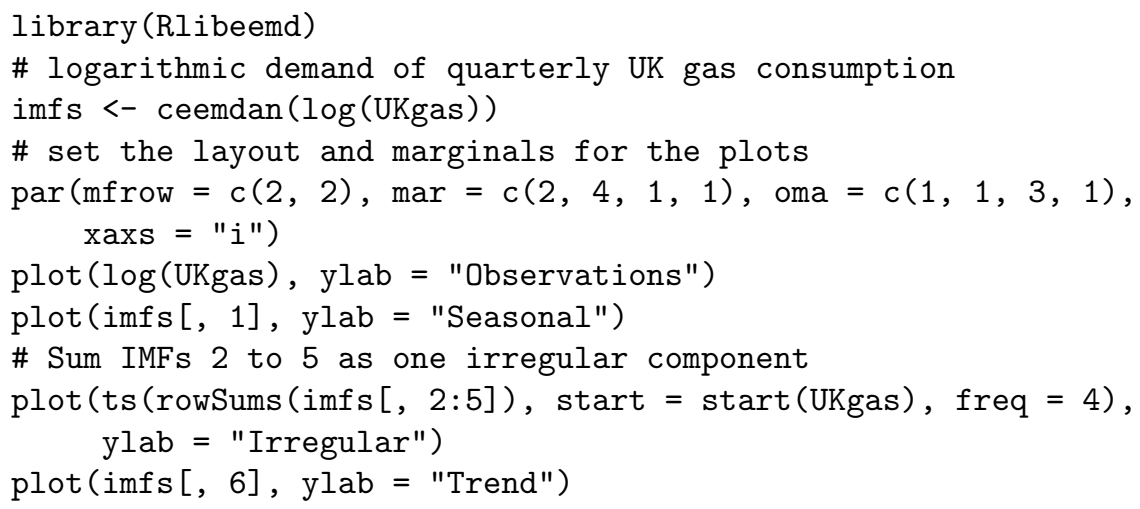

CEEMDAN extracts five IMFs and the residual, which captures the underlying trend. The first IMF contains the seasonal effect whereas rest of the IMFs contain the irregular part. In the example code, the IMFs number 2 to 5 are combined by taking the sum over the IMFs at each time point. The resulting irregular component is then defined as a time series object for proper plotting behavior. The resulting plot can be seen in Fig. 3. More examples can be found from the documentation distributed with the Rlibeemd package.

\footnotetext{
2 http://cran.r-project.org/web/packages/Rlibeemd/index.html

3 https://github.com/helske/Rlibeemd
} 

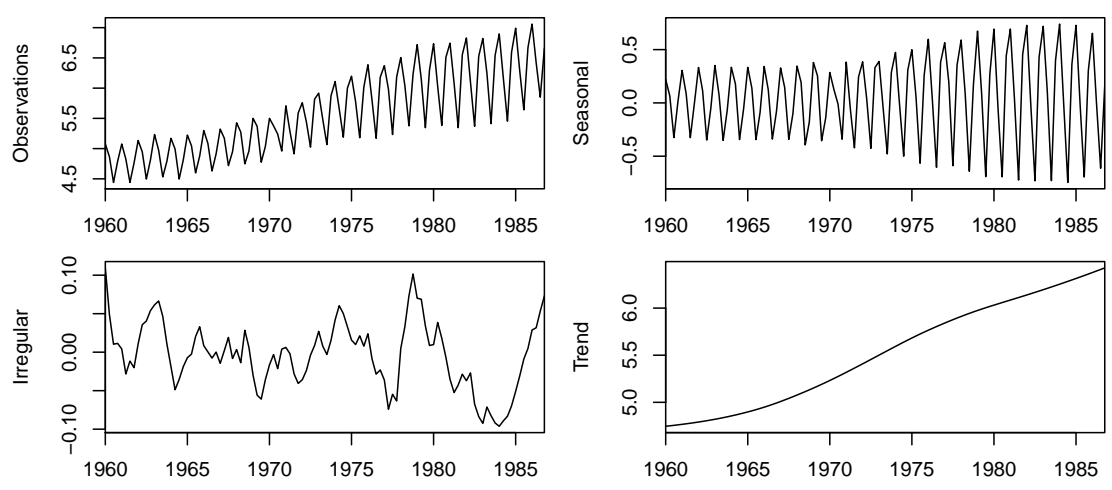

Fig. 3 Example plot produced using Rlibeemd, showing the various components extracted from UK gas consumption with CEEMDAN.

\section{Comparison to other implementations}

Several established EMD, EEMD and CEEMDAN implementations are publicly available. Of these the most commonly used ones are the reference EEMD implementation by Wu and Huang (2009), the EMD toolbox by P. Flandrin's group $^{4}$, the original CEEMDAN implementation by Torres et al (2011) (also available from $\mathrm{P}$. Flandrin's home page), the EMD R package by Kim and Oh (2009), and the hht R package by Bowman and Lees (2014) containing EEMD and CEEMDAN implementations. There is also a rudimentary Python implementation of EMD/EEMD included in the Python Time Series Analysis package $(\mathrm{PTSA})^{5}$ by the Ohio State University Computational Memory Lab. We are also aware of an EEMD code for Matlab by Wang et al (2014), but it is only available in binary form. While the algorithms implemented in libeemd are implemented also by others (with possible minor variations such as discussed in Sec. 2.2), our implementation brings several benefits, which we outline in the following.

Except the packages written for $\mathrm{R}$ and the PTSA, all other implementations mentioned here are written for Matlab. It is very difficult to use routines written in Matlab from other languages, and using Matlab requires the purchase of a license. Likewise, $\mathrm{R}$ codes are mostly only usable on $\mathrm{R}$, and Python codes in projects using Python (some commercial statistical software such as SAS and SPSS support R or Python plug-ins). Having a stable C library is very useful for expanding the user base of EMD, since almost every high-level language uses $\mathrm{C}$ as the low-level language for writing extension modules. The $\mathrm{C}$ interface of libeemd has been designed to make the work of interface builders

\footnotetext{
4 http://perso.ens-lyon.fr/patrick.flandrin/emd.html

5 http://ptsa.sourceforge.net
} 
as easy as possible by only using standard $\mathrm{C}$ data types in the public interface. Having the algorithms written in a low-level language such as $\mathrm{C}$ also brings immediate and substantial speed improvements over implementations using only interpreted languages.

We would also like to highlight the importance of clearly written, welldocumented and thoroughly tested code, as well as modern programming practices and proper version control. We have taken effort in making the code of libeemd easily readable and modifiable by others, making it easier for other people to use libeemd as a basis for not only new applications of EMD, but also new variants of the underlying algorithms. The source code of libeemd is hosted on Bitbucket ${ }^{6}$, making it easy for users to track changes in the program, report issues, and discuss and publish improvements to the program. Being published under an open-source license, libeemd protects the users' right to modify the code, fostering the development of better software.

Besides the original CEEMDAN implementation and the $\mathrm{R}$ package hht, to our knowledge there are currently no other publicly available implementations of CEEMDAN. Our implementation thus also provides the first $\mathrm{C}$ and Python implementations of CEEMDAN, as well as the first parallelized CEEMDAN implementation.

There are also implementations that focus on computing EMD-like decompositions in real time from measurement data, often focusing on specific kinds of data and using specialized hardware and/or GPU acceleration. Our implementation focuses on providing a free and generic software implementation of the EMD algorithms for offline data analysis, so we have not included these implementations in our comparison.

\subsection{Performance comparison}

As mentioned before, writing the core algorithm in a low-level language brings a substantial improvement in numerical performance. To provide an example in the case of $R$, we compare briefly the performance of the $R$ package hht (Bowman and Lees, 2014) and our Rlibeemd. As input data, we use ECG (electrocardiogram) data from the MIT-BIH Normal Sinus Rhythm Database ${ }^{7}$, which was also used in the original article of Torres et al (2011). We use a value of 0.2 as the relative standard error of added noise, and the S-number stopping criterion with $S=4$ together with a maximum number of siftings of 50. Length of the signal is varied from 100 to 2000 , and ensemble size is varied from 50 to 500. The maximum number of IMFs to extract (including the residual) is set to six.

Benchmarking was performed on a system with an Intel Quad-Core i7-4770 $3.40 \mathrm{GHz}$ CPU and $16 \mathrm{~GB}$ of RAM running on 64 bit Microsoft Windows 7 Enterprise platform. Figure 4 shows how the required computation time for CEEMDAN scales with the number of data points. Note that the $y$-axis is

\footnotetext{
6 https://bitbucket.org/luukko/libeemd

7 http://www.physionet.org/cgi-bin/atm/ATM
} 


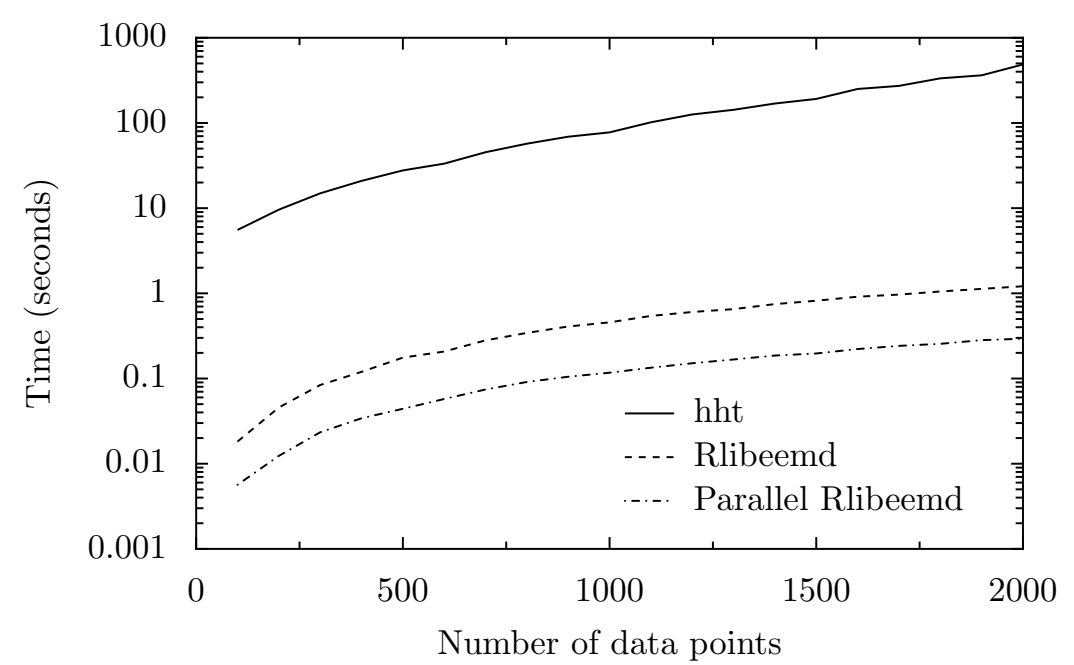

Fig. 4 Performance comparison of the $\mathrm{R}$ packages hht (function CEEMD) and Rlibeemd (function ceemdan) for varying number of input data points. The y-axis shows the mean computation time required to run the CEEMDAN decomposition function. The implementation from Rlibeemd is approximately two orders of magnitude faster than hht, and it can be sped up further with parallelization.

logarithmic in order to deal with the different scales of performance. The performance of Rlibeemd is clearly superior to hht, which is approximately two orders of magnitude slower than non-parallerized Rlibeemd. The parallerized version of Rlibeemd is approximately four times faster than the nonparallerized Rlibeemd, which is the expected speedup for a quad-core CPU. Similar results were obtained also when the ensemble size was varied instead of the number of data points, and when comparing results for the EEMD algorithm.

It should be noted that in addition to extracting the IMFs, the CEEMD function of hht also performs a Hilbert transform on the IMFs in order to obtain their instantaneous frequencies. However, this additional step should have only a marginal effect to overall computational time, as the Hilbert transform only needs to be executed once for the final IMFs.

We also compared the performance of Rlibeemd and the R package EMD (Kim and Oh, 2009) in performing the ordinary EMD decomposition. With the same overall parameters as the CEEMDAN comparison, the EMD package was found to be approximately three orders of magnitude slower than Rlibeemd.

\section{Conclusions}

We have presented a free software code library which implements the ensemble empirical mode decomposition (EEMD), of which the regular empirical mode decomposition (EMD) is a special case, and its complete variant CEEMDAN. 
Since our library is implemented in C, it can be readily interfaced with a variety of high-level languages for inclusion into existing data analysis software. As an example of this we have provided complete Python and $\mathrm{R}$ interfaces. By implementing the algorithm in $\mathrm{C}$ and providing interfaces to high-level languages the implementation retains the unmatched speed of a low-level language while gaining the ease of use and flexibility of higher level languages. Our implementation corrects a minor issue with equal data points in the reference EEMD implementation by Wu and Huang (2009), includes the first optimized and parallel implementation of CEEMDAN, and provides a solid and well-documented basis for existing and future improvements of the (E)EMD method.

Acknowledgements This work was supported by the Finnish Cultural Foundation, the Emil Aaltonen Foundation, the Academy of Finland, and the European Community's FP7 through the CRONOS project, Grant Agreement No. 280879. The authors wish to thank N. E. Huang for useful discussions.

\section{References}

Bowman DC, Lees JM (2014) The Hilbert-Huang Transform: Tools and Methods. R version 3.1.0 (2014-04-10)

Chang LW, Lo MT, Anssari N, Hsu KH, Huang N, Hwu WM (2011) Parallel implementation of multi-dimensional ensemble empirical mode decomposition. In: Acoustics, Speech and Signal Processing (ICASSP), 2011 IEEE International Conference on, pp 1621-1624

Chen Q, Huang N, Riemenschneider S, Xu Y (2006) A B-spline approach for empirical mode decompositions. Adv Comput Math 24:171-195

Colominas MA, Schlotthauer G, Torres ME, Flandrin P (2012) Noise-assisted EMD methods in action. Adv Adapt Data Anal 04:1250,025

Dätig M, Schlurmann T (2004) Performance and limitations of the HilbertHuang transformation (HHT) with an application to irregular water waves. Ocean Eng 31:1783-1834

Eddelbuettel D (2013) Seamless R and C++ Integration with Rcpp. Springer, New York

Eddelbuettel D, François R (2011) Rcpp: Seamless R and C++ integration. Journal of Statistical Software 40(8):1-18, URL http://www.jstatsoft. org/v40/i08/

Engeln-Müllges G, Uhlig F (1996) Numerical Algorithms With C. SpringerVerlag Berlin Heidelberg

Galassi M, Davies J, Theiler J, Gough B, Jungman G, Alken P, Booth M, Rossi F (2009) GNU Scientific Library Reference Manual. Network Theory Limited, UK, URL http://www.gnu.org/software/gsl/

Huang H, Pan J (2006) Speech pitch determination based on Hilbert-Huang transform. Signal Process 86:792-803 
Huang N, Shen S (2005) The Hilbert-Huang transform and its applications. Interdisciplinary Mathematical Sciences, World Scientific Publishing Company, Inc.

Huang NE, Wu Z (2008) A review on Hilbert-Huang transform: method and its applications to geophysical studies. Rev Geophys 46

Huang NE, Shen Z, Long SR, Wu MC, Shih HH, Zheng Q, Yen NC, Tung CC, Liu HH (1998) The empirical mode decomposition and the Hilbert spectrum for nonlinear and non-stationary time series analysis. P Roy Soc Lond A Mat 454:903-995

Huang NE, Shen Z, Long SR (1999) A new view of nonlinear water waves: The Hilbert spectrum. Annu Rev Fluid Mech 31:417-457

Huang NE, Wu MLC, Long SR, Shen SSP, Qu W, Gloersen P, Fan KL (2003) A confidence limit for the empirical mode decomposition and Hilbert spectral analysis. P Roy Soc Lond A Mat 459:2317-2345

Kim D, Oh HS (2009) EMD: A package for empirical mode decomposition and Hilbert spectrum. The R Journal 1:40-46

Morales IO, Landa E, Stránský P, Frank A (2011) Improved unfolding by detrending of statistical fluctuations in quantum spectra. Phys Rev E 84:016,203-016,209

Oliphant TE (2007) Python for scientific computing. Comput Sci Eng 9:10-20

Pachori RB, Bajaj V (2011) Analysis of normal and epileptic seizure EEG signals using empirical mode decomposition. Comput Meth Prog Bio 104:373381

Shulin L, Haifeng Z, Hui W, Rui M (2007) Application of improved EMD algorithm for the fault diagnosis of reciprocating pump valves with spring failure. In: Signal Processing and Its Applications (ISSPA) 2007. 9th International Symposium on, pp 1-4

Torres ME, Colominas MA, Schlotthauer G, Flandrin P (2011) A complete ensemble empirical mode decomposition with adaptive noise. In: Acoustics, Speech and Signal Processing (ICASSP), 2011 IEEE International Conference on, pp 4144-4147

Wang YH, Yeh CH, Young HWV, Hu K, Lo MT (2014) On the computational complexity of the empirical mode decomposition algorithm. Physica A 400:159-167

Wu Z, Huang NE (2004) A study of the characteristics of white noise using the empirical mode decomposition method. P Roy Soc Lond A Mat 460:15971611

Wu Z, Huang NE (2009) Ensemble empirical mode decomposition: a noiseassisted data analysis method. Adv Adapt Data Anal 01:1-41

Wu Z, Huang NE, Long SR, Peng CK (2007) On the trend, detrending, and variability of nonlinear and nonstationary time series. P Natl Acad Sci USA $104: 14,889-14,894$

Zhang X, Lai KK, Wang SY (2008) A new approach for crude oil price analysis based on empirical mode decomposition. Energ Econ 30:905-918 